\title{
Contrast imaging and gene delivery through the combined use of novel cationic liposomal microbubbles and ultrasound in rat carotid arteries
}

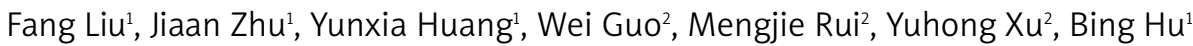

\author{
1Shanghai Jiaotong University Affiliated Sixth People's Hospital, Shanghai Institute \\ of Ultrasound in Medicine, China \\ 2Pharmacy School of Shanghai Jiaotong University, China
}

Submitted: 14 February 2012

Accepted: 20 May 2012

Arch Med Sci 2013; 9, 2: 347-353

DOI: $10.5114 /$ aoms.2013.34536

Copyright $\odot 2013$ Termedia \& Banach

\begin{abstract}
Introduction: Lipid-coated cationic microbubbles represent a new class of agents with both diagnostic and therapeutic applications. The main goal of this study was to evaluate the efficiency of gene transfer through the combined use of microbubbles and ultrasound in rat carotid arteries. Furthermore, we assessed whether the cationic liposomal microbubbles could allow long-term enhanced imaging, comparing with SonoVue ${ }^{\circledR}$.

Material and methods: Normal rat carotid arteries were imaged after intravenous bolus injections of $0.5 \mathrm{ml} / \mathrm{kg}$ of two contrast agents (SonoVue ${ }^{\circledR}$ and the cationic liposomal microbubbles). Forty Sprague-Dawley rats were divided into 5 groups according to ultrasound parameters and were treated with or without microbubbles. All rats were sacrificed after being transfected for 2 days. The level of protein expression was determined by western blot analysis.

Results: The enhancing time of self-made microbubbles was much longer than that of SonoVue ${ }^{\circledR}$ in rat carotid arteries $(p<0.05)$. The results of the western blot analysis revealed that the expression of SR-BI DNA in the carotid artery was highest in the SR-BI + US/CLM group $(p<0.05)$.

Conclusions: These results suggest that the novel cationic liposomal microbubbles enhance image quality over a longer period than does SonoVue ${ }^{\circledR}$. Additionally, the combination of ultrasound and this new type of microbubble can act synergistically to increase SR-BI DNA transfection.
\end{abstract}

Key words: microbubbles, contrast-enhanced ultrasound, cationic liposomal microbubble, gene delivery, rat.

\section{Introduction}

Atherosclerosis is known to be a disease which results in a host of complications of cardiovascular disease, including ischemia, acute coronary syndromes and stroke. Nowadays, drug therapy is the main method for the treatment of atherosclerosis [1]. Recent progress in gene therapy has led to the development of a new treatment strategy for cardiovascular diseases. Viral and non-viral vectors have been used for DNA delivery with varying levels of success, but have always been accompanied by disadvantages, such as insufficient expression levels or safety concerns [2, 3].

Compared with viral transfection techniques, microbubble-mediated ultrasound (US) has several advantages as a novel non-viral genetic carri-

\author{
Corresponding author: \\ Prof. Jiaan Zhu MD, PhD \\ Shanghai Jiaotong University \\ Affiliated Sixth People's \\ Hospital \\ Shanghai Institute \\ of Ultrasound in Medicine \\ 600 Yishan Road \\ 200233 Shanghai, China \\ Phone: +86-21-64369181 \\ E-mail: zhuja@sjtu.edu.cn
}


er. (1) Visibility: the US reflectivity of microbubbles allows for image-guided gene delivery [4]. (2) Safety: cavitation caused by US-triggered destruction of the microbubbles transiently and reversibly increases the permeability of cells and tissues without affecting cell activity; in addition, microbubbles do not exhibit cytotoxicity or immunogenicity $[5,6]$. (3) Manufacturing ease: unlike viral carriers, microbubbles can be used on a large scale. (4) Site-specificity: using microbubbles, plasmids can be administered either intravenously or directly into tissues of interest. Gene delivery can then be targeted to a specific area that is subsequently exposed to US, thus avoiding delivery to non-target cells.

However, microbubbles are generally unstable, and their mean diameter of approximately 1-6 $\mu \mathrm{m}$ is too large for intravascular applications [7]. Moreover, it is difficult to modify functional particles such as targeting molecules to fit on the surfaces of microbubbles. Therefore, microbubbles should generally be smaller than red blood cells and stable after injection into the blood. Furthermore, their surfaces should be easily modifiable with functional molecules for targeting.

In this study, we developed a novel lipid-coated cationic microbubble. The novel cationic liposomal microbubble (CLM) was composed of perfluoropropane gas encapsulated in a phospholipid shell. We attempted to evaluate the efficiency of gene transfer using US in rat carotid arteries. Furthermore, we assessed whether the microbubbles can pass through the pulmonary circulation repeatedly and whether they can enhance imaging quality over the long term compared with results achieved using SonoVue ${ }^{\circledR}$.

\section{Material and methods}

\section{Plasmid purification and microbubble preparation}

Full-length cDNAs of rabbit Scavenger receptor class B member 1 (SR-BI) were subcloned into vector pcDNA3.1 (Invitrogen). Plasmid DNA amplification was performed using the QIAGEN plasmid Giga Kit (Clontech, Palo Alto, CA).

Cationic liposomes composed of 1,2-dipalmitoylsn-glycero-3-phosphocholine (DPPC) (NOF Corporation, Tokyo, Japan), 1,2-distearoyl-sn-glycero-3phosphoethanolamine- $\mathrm{N}$-[methoxy(polyethylene glycol)-2000] (DSPE-PEG2000) (Avanti, Germany) and 1,2-dioleoyl-3-trimethyl-ammonium-propane (DOTAP) [94 : $6: 100(\mathrm{~m} / \mathrm{m})]$ were prepared using the thin-film dispersion method. The mixed solution consisting of PBS, propylene glycol and glycerin with volume ratio of $8: 1: 1$ was applied as a thin layer in a rotary evaporation bottle and was sonicated while sparging perfluoropropane. The particle size of the microbubbles was measured using a Zetasizer Nano S (Malvern Instruments, UK) after the samples were diluted with water.

SR-BI DNA was simply added to the pre-formed microbubbles and gently agitated. Then SR-BI DNA was combined with CLMs by electrostatic charge coupling.

\section{Assessment of plasmid-microbubble conjugation}

A combination of SR-BI DNA and CLMs was incubated for $10 \mathrm{~min}$ with various mole ratios $(2: 1$, $1: 1,1: 2,1: 3,1: 4,1: 5,1: 6,1: 7,1: 8)$. Gel electrophoresis was used to demonstrate plasmidmicrobubble coupling. DNA was visualized by fluorescence under UV irradiation. A propidium iodide (PI) stain was used to detect the location of the plasmid on the microbubbles [8].

\section{Damage to plasmid DNA caused by CLM and US}

To investigate the integrity of the plasmid after sonication, plasmid DNA (SR-BI; $1 \mu \mathrm{g}$ ) dissolved in $500 \mu \mathrm{l}$ of Opti-MEM (Invitrogen Corporation, Carlsbad, CA) was exposed to US with or without CLMs $(15 \mu \mathrm{l})$ under the following conditions: frequency: $1 \mathrm{MHz}$; duty: 50\%; intensity: 0, 1.0, 2.1, 3.2 W/ $\mathrm{cm}^{2}$; time: $0,10,30 \mathrm{~s}$. We used naked plasmid DNA as a control. In these groups, plasmid DNA and CLMs were placed in contact with one another for $10 \mathrm{~min}$. Cationic liposomal microbubbles were then removed using phenol/chloroform and plasmid DNA recovered by ethanol precipitation was dissolved in TE buffer and resolved by electrophoresis in $0.7 \%$ agarose gels $[9,10]$.

\section{Experimental animals}

Fifty male Sprague-Dawley rats weighing $250 \mathrm{~g}$ to $300 \mathrm{~g}$ were used. The rats were supplied by the experimental animal center of Shanghai Jiaotong University Affiliated Shanghai Sixth People's Hospital. This experiment was approved by the Animal Care and Use Committee of Shanghai Jiaotong University Affiliated Shanghai Sixth People's Hospital.

\section{US contrast imaging after the intravenous injection of microbubbles}

Ten rats were anesthetized with $4 \%$ pentobarbital sodium ( $1 \mathrm{ml} / \mathrm{kg}$ ) by intraperitoneal injection, laid on their backs and restrained on the experimental table. Before the experiments, the femoral vein was catheterized and the hair over the neck was removed with depilatory cream.

An ultrasonic diagnostic instrument (Mylab 90, Esaote, Genoa, Italy) was employed using $13-\mathrm{MHz}$ linear-array transducers. As the carotid artery was 
depicted clearly, all the conditions mentioned above were kept consistent during the contrast imaging.

Two types of microbubbles, SonoVue ${ }^{\circledR}$ (Bracco Research, Geneva, Switzerland) and the novel CLMs, were injected via the femoral vein. Referring to a previous study, the SonoVue ${ }^{\circledR}$ powder was dissolved in $5 \mathrm{ml}$ of physiological saline. Each type of microbubble was administered $0.5 \mathrm{ml} / \mathrm{kg}$ [11]. The microbubbles were injected via the femoral vein, followed by normal saline to wash the tube. The bolus injection of microbubbles was flushed into the circulation of the animal and was favorable for improving the signal-to-noise imaging ratio. Realtime imaging of the carotid artery was observed for $3 \mathrm{~min} 30 \mathrm{~s}$ after the injection. The two-dimensional images were saved prior to injection.

\section{In vivo gene delivery into rat carotid arteries}

Forty rats were anesthetized and fixed as described above. Cationic liposomal microbubbles were injected slowly via the femoral vein within the specified time period.

Gene delivery was then performed according to the assigned treatment groups: control group - no treatment; SR-BI group - local injection of SRBI $(100 \mu \mathrm{g}) ; \mathrm{SR}-\mathrm{BI}+\mathrm{US} / \mathrm{SonoVue}{ }^{\circledR}$ group - Intravenous push of SRBI $(100 \mu \mathrm{g})$ and SonoVue ${ }^{\circledR}(1 \mathrm{ml})$ by US; SR-BI + CLM group - Intravenous push of SRBI $(100 \mu \mathrm{g})$ and CLM (1 ml); SR-BI + US/CLM group Intravenous push of SRBI $(100 \mu \mathrm{g})$ and CLM (1 ml) by US ( $n=8$ per group). The US parameters were performed as follows: frequency: $1 \mathrm{MHz}$, duty: $50 \%$; intensity: $2.0 \mathrm{~W} / \mathrm{cm}^{2}$, time: $15 \mathrm{~min}$. All rats were sacrificed after being transfected for 2 days. Tissue was obtained for western blot analysis from the US imaged rat carotid arteries. Western blot analysis was performed as described previously [12].

\section{Statistical analysis}

All data were presented as the mean SD. Preand post-contrast of carotid artery's grey scale data were compared by Student's t-test in SPSS 16.0 program. Multiple comparisons among the groups were performed with a one-way ANOVA analysis. Statistical significance was set at $p<0.05$ for all of the tests.

\section{Results}

\section{CLM and plasmid conjugation}

The diameter range of the CLM was $0.71 \mu \mathrm{m}$ to $5.56 \mu \mathrm{m}$, the mean diameter was $1.31 \mu \mathrm{m}$ and the concentration was $(3.12 \pm 0.39) \times 10^{9} / \mathrm{ml}$. Figure 1 shows a typical image of CLMs as visualized by optical microscopy.

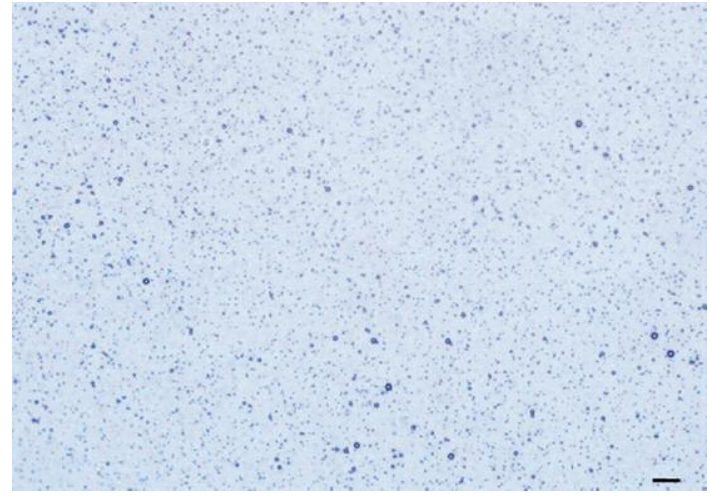

Figure 1. Typical images of cationic liposomal microbubbles from optical microscopy

The scale bar is $20 \mu \mathrm{m}$ (original magnification: 40x)

$\mathrm{SR}-\mathrm{BI} / \mathrm{CLM}$

Control 2:1 $1: 1 \quad 1: 2 \quad 1: 3 \quad 1: 4 \quad 1: 5 \quad 1: 6 \quad 1: 7 \quad 1: 8$

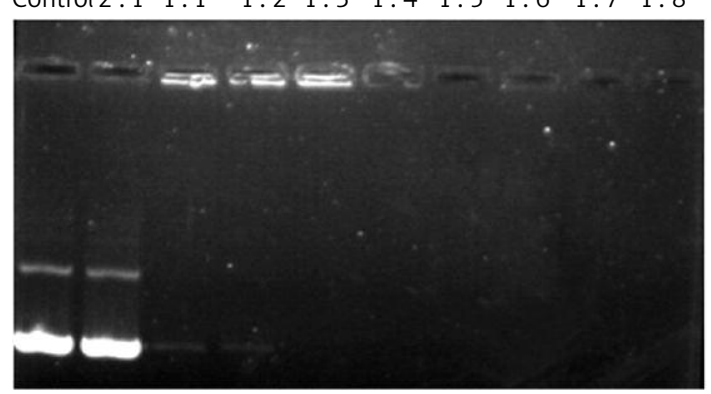

Figure 2. The mobility of SR-BI/CLM complexes, as analyzed by agarose gel electrophoresis

$S R-B I$ - scavenger receptor class B member 1, CLM - cationic liposomal microbubble

SR-BI was mixed with CLMs in different molar ratios to form plasmid-microbubble complexes, and the effect of conjugation was investigated by agarose gel electrophoresis. Figure 2 shows that the optimal molar ratio of the plasmid-microbubble complexes was $1: 3$. Incorporation of the plasmid into the phospholipid shell of the microbubbles was also confirmed by confocal microscopy using the PI staining method (Figure 3).

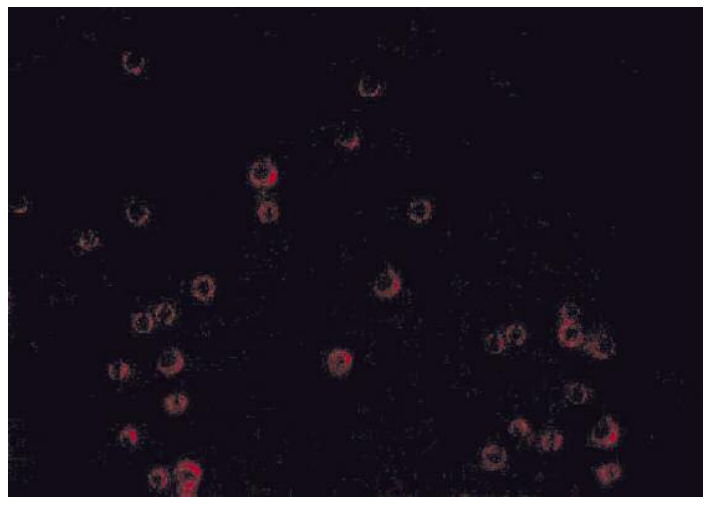

Figure 3. The ultrasound contrast agent loaded with SR-BI under fluorescence microscopy (original magnification: 400x). The shell of the microbubble erupted with red fluorescence after SR-BI was dyed with PI $S R-B I-S c a v e n g e r$ receptor class $B$ member $1, P I$ - propidium iodide 


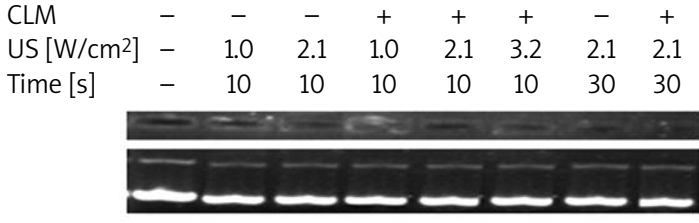

Figure 4. Stability of the plasmid DNA under various conditions of ultrasound with or without cationic liposomal microbubbles

\section{Stability of CLMs/SR-BI complexes after sonication}

Heat and jet streams are generally induced with cavitation, which can damage plasmid DNA. We sought to assess the stability of SR-BI after US with or without CLMs using agarose gel electrophoresis (Figure 4). The presence of unchanged bands indicated that the plasmid DNA remained intact. The results demonstrated that 10-30 s of US did not degrade plasmid DNA regardless of the presence or absence of CLMs.

\section{US contrast imaging of carotid arteries}

One of 10 rats died due to an anesthetic accident. Both SonoVue ${ }^{\circledR}$ and the CLM markedly en-
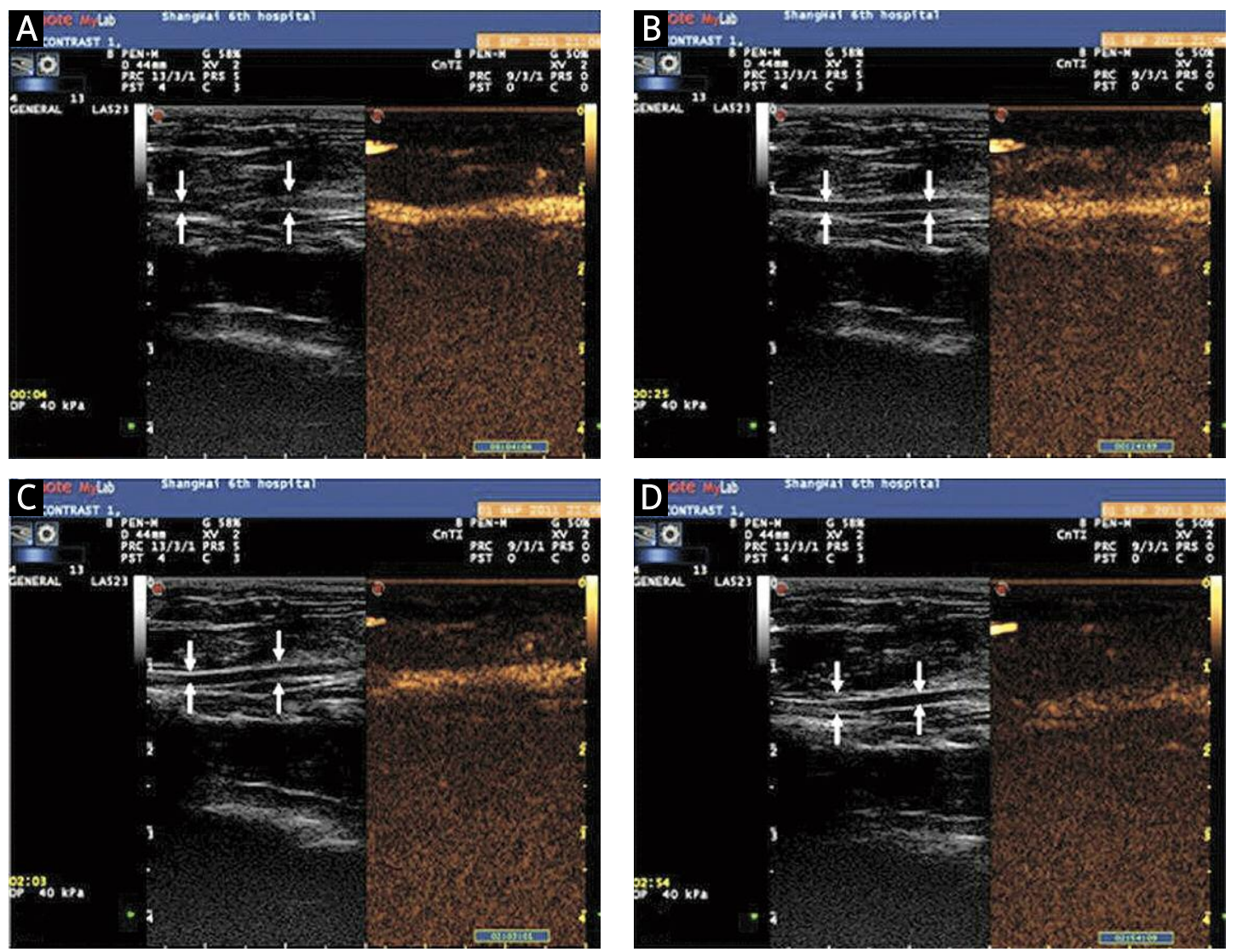

Figure 5. Contrast-enhanced imaging of the carotid artery by the intravenous injection of cationic liposomal microbubbles (arrow indicates the carotid artery): A - images of the carotid artery at 4 s after CLM injection; B - images of the carotid artery at $25 \mathrm{~s}$ after CLM injection; $\mathbf{C}$ - images of the carotid artery at $123 \mathrm{~s}$ after CLM injection; D - images of the carotid artery at $174 \mathrm{~s}$ after CLM injection CLM - cationic liposomal microbubble 

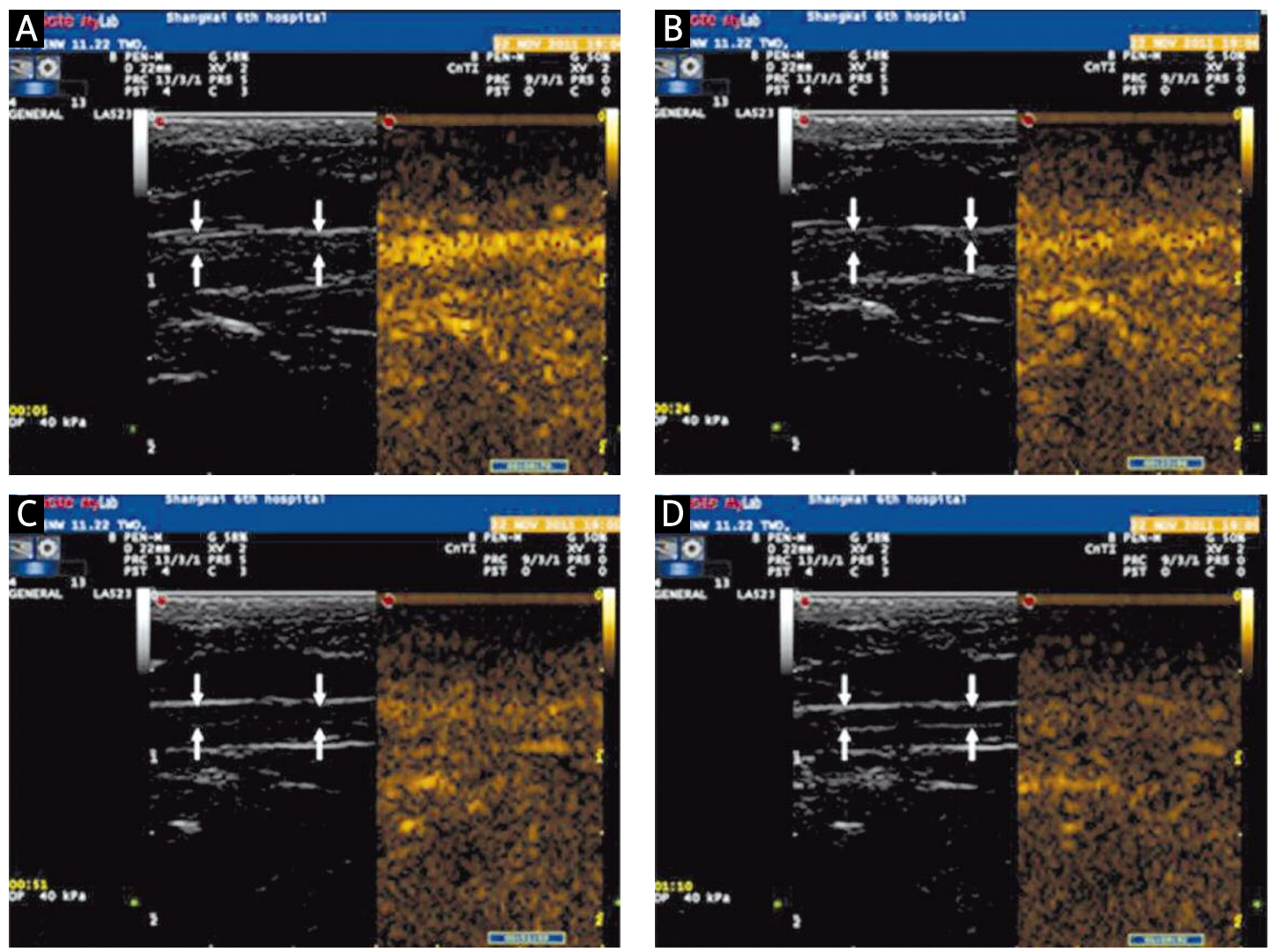

Figure 6. Contrast-enhanced imaging of the carotid artery by the intravenous injection of SonoVue (arrow indicates the carotid artery): A - images of the carotid artery at $4 \mathrm{~s}$ after SonoVue injection; B - images of the carotid artery at $25 \mathrm{~s}$ after SonoVue injection; C - images of the carotid artery at $123 \mathrm{~s}$ after SonoVue injection; D - images of the carotid artery at $174 \mathrm{~s}$ after SonoVue injection

Meanwhile, the transfection of SR-BI DNA by means of ultrasound with CLMs was higher than that by means of ultrasound with SonoVue ${ }^{\circledR}$. However, there was no statistical significance compared with the two groups $(p>0.05)$.

\section{Discussion}

Microbubbles are used as US contrast agents to enhance the visualization of blood flow in the human body. Recently, the development of site targeted microbubbles opened up the possibility of gene delivery. SonoVue ${ }^{\circledR}$, as a second-generation US contrast agent consisting of phospholipid-stabilized microbubbles filled with sulfur hexafluoride, has been widely used for diagnosis in clinical settings. The size distribution of SonoVue ${ }^{\circledR}$ ranges from $1 \mu \mathrm{m}$ to $12 \mu \mathrm{m}$, yielding a total volume of $2 \times 10^{8} / \mathrm{ml}$ [13]. However, it also has a disadvantage: the short duration of enhancement in the blood. In this study, the duration of enhancement of SonoVue ${ }^{\circledR}(70 \mathrm{~s})$ is much shorter than that achieved by CLM (180 s). One possible explanation is that CLMs caused a long, steady enhancement in normal carotid artery contrast imaging as follows: PEG, one of the components of CLM, not only can stabilize the surface of conventional liposomes but also decreases

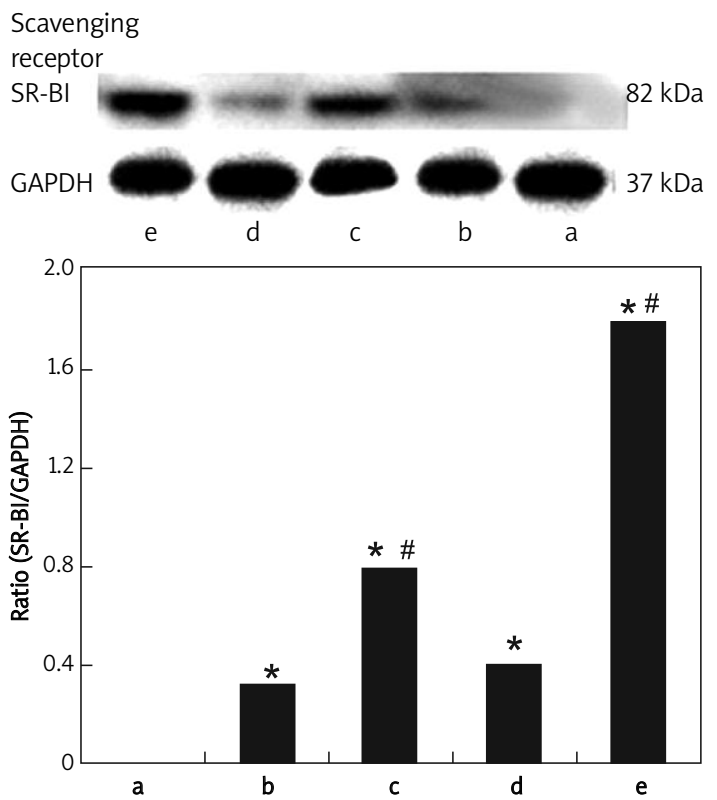

Figure 7. Expression of SR-BI in each group by western blot analysis. GAPDH served as the reference. The bar graphs show the ratio of SR-BI to GAPDH Data represent the mean $\pm S D$ for groups of 8 rats; ${ }^{*} p<0.01$ vs. the control group, ${ }^{\#} p<0.05$ vs. the SR-BI group 
the degree of immunogenicity $[9,14]$. Furthermore, CLMs consist of perfluoropropane, which has a higher molecular weight; CLMs have a correspondingly longer residence time in the blood [9]. Therefore, our microbubble exhibits good stability in the internal circulation. That is to say, it may have a longer metabolic half-life than its SonoVue ${ }^{\circledR}$ counterpart.

In this study, the combination of US and microbubbles was able to effectively transduce plasmid DNA into the carotid artery. This result suggested that bubble liposomes could quickly transduce plasmid DNA into the artery by cavitation even with brief contact between bubble liposomes and the endothelial cells. The results achieved in the SR-BI $+C L M$ and $S R-B I+U S / C L M$ groups demonstrate that we successfully delivered SR-BI to specific areas by local exposure to US. Thus, gene expression depended on the site of the US. Furthermore, the transfection efficiency in the SR-BI + US/CLM group was higher than in the SR-BI + US/ SonoVue ${ }^{\circledR}$ group. This may be because CLMs were more stable in the blood than were the SonoVue ${ }^{\circledR}$ microbubbles. Another possible reason is that SR-BI was mixed with CLMs during the electrostatic charge coupling. Compared with the combination method that relies on simple shaking, this conjugation method may load more plasmid DNA stably. In the future, we would like to evaluate whether the gene carrier rate affects the efficiency of gene transfer.

However, toxicity is still an obstacle to the application of cationic lipids for gene therapy in vitro and in vivo $[15,16]$. In our study, one rat died during the intravenous injection of CLMs and US. A possible reason is that long-term existence of CLMs in blood may influence the rat organs and tissues. Meanwhile, further studies are still needed on the safety of CLMs in vivo.

Preliminary studies into the utility of US for gene delivery used frequencies in the range of $20-50 \mathrm{kHz}$ $[17,18]$. However, these frequencies are also known to induce tissue damage and cavitation if not properly controlled [19-21]. To overcome this problem, several studies have used frequencies of $1-3 \mathrm{MHz}$, intensities of $0.5-2 \mathrm{~W} / \mathrm{cm}^{2}$, and pulse-modulation [22-25]. In our study, we employed similar US parameters (frequency: $1 \mathrm{MHz}$; duty: $50 \%$; intensity: $2 \mathrm{~W} / \mathrm{cm}^{2}$; time: $15 \mathrm{~min}$ ), which did not induce irreversible tissue damage. However, as this is preliminary research, further studies are needed to optimize the US parameters.

In conclusion, these novel cationic liposomal microbubbles can enhance the US imaging of rat carotid arteries and can achieve a longer enhancement time than do the SonoVue ${ }^{\circledR}$ microbubbles. Moreover, the combination of US and these novel microbubbles can have a synergistic effect in increasing DNA transfection. Thus, CLM may be- come a new agent for both diagnostic and therapeutic applications.

\section{Acknowledgments}

This work was supported by the National Natural Science Foundation of China (30970794) and the Natural Science Foundation of Shanghai Science and Technology Commission (09ZR1424300).

\section{References}

1. Mercando AD, Lai HM, Aronow WS, et al. Reduction in atherosclerotic events: a retrospective study in an outpatient cardiology practice. Arch Med Sci 2012; 8: 57-62.

2. Rolland AP. From genes to gene medicines: recent advances in nonviral gene delivery. Crit Rev Ther Drug Carrier Syst 1998; 15: 143-98.

3. Iwamoto HS, Trapnell BC, McConnell CJ, Daugherty C, Whitsett JA. Pulmonary inflammation associated with repeated, prenatal exposure to an E1, E3-deleted adenoviral vector in sheep. Gene Ther 1999; 6: 98-106.

4. Huang SL. Liposomes in ultrasonic drug and gene delivery. Adv Drug Deliv Rev 2008; 60: 1167-76.

5. Mayer CR, Bekeredjian R. Ultrasonic gene and drug delivery to the cardiovascular system. Adv Drug Deliv Rev 2008; 60: 1177-92.

6. Bekeredjian R, Kroll RD, Fein E, et al. Ultrasound targeted microbubble destruction increases capillary permeability in hepatomas. Ultrasound Med Biol 2007; 33: 1592-8.

7. Lindner JR. Microbubbles in medical imaging: current applications and future directions. Nat Rev Drug Discov 2004; 3: 527-32.

8. Li X, Wang Z, Ran H, et al. Experimental research on therapeutic angiogenesis induced by hepatocyte growth factor directed by ultrasound-targeted microbubble destruction in rats. J Ultrasound Med 2008; 27: 453-60.

9. Suzuki R, Takizawa T, Negishi Y, et al. Gene delivery by combination of novel liposomal bubbles with perfluoropropane and ultrasound. J Control Release 2007; 117: 130-6.

10. Deshpande MC, Prausnitz MR. Synergistic effect of ultrasound and PEI on DNA transfection in vitro. J Control Release 2007; 118: 126-35.

11. Liu P, Wang X, Zhou S, Hua X, Liu Z, Gao Y. Effects of a novel ultrasound contrast agent with long persistence on right ventricular pressure: comparison with SonoVue. Ultrasonics 2011; 51: 210-4.

12. Ritsch A, Tancevski I, Schgoer W, et al. Molecular characterization of rabbit scavenger receptor class $B$ types I and II: portal to central vein gradient of expression in the liver. J Lipid Res 2004; 45: 214-22.

13. Schneider M, Arditi M, Barrau MB, et al. BR1: a new ultrasonographic contrast agent based on sulfur hexafluoride-filled microbubbles. Invest Radiol 1995; 30: 451-7.

14. Unger EC, Porter T, Culp W, Labell R, Matsunaga T, Zutshi R. Therapeutic applications of lipid-coated microbubbles. Adv Drug Deliv Rev 2004; 56: 1291-314.

15. Sakurai F, Nishioka T, Saito H, et al. Interaction between DNA-cationic liposome complexes and erythrocytes is an important factor in systemic gene transfer via the intravenous route in mice: the role of the neutral helper lipid. Gene Ther 2001; 8: 677-86.

16. Filion MC, Phillips NC. Toxicity and immunomodulatory activity of liposomal vectors formulated with cationic lipids 
toward immune effector cells. Biochim Biophys Acta 1997; 1329: 345-56.

17. Fechheimer M, Boylan JF, Parker S, Sisken JE, Patel GL, Zimmer SG. Transfection of mammalian cells with plasmid DNA by scrape loading and sonication loading. Proc Natl Acad Sci U S A 1987; 84: 8463-7.

18. Joersbo M, Brunstedt J. Protein synthesis stimulated in sonicated sugar beet cells and protoplasts. Ultrasound Med Biol 1990; 16: 719-24.

19. Guzmán HR, McNamara AJ, Nguyen DX, Prausnitz MR. Bioeffects caused by changes in acoustic cavitation bubble density and cell concentration: a unified explanation based on cell-to-bubble ratio and blast radius. Ultrasound Med Biol 2003; 29: 1211-22.

20. Miller DL, Pislaru SV, Greenleaf JE. Sonoporation: mechanical DNA delivery by ultrasonic cavitation. Somat Cell Mol Genet 2002; 27: 115-34.

21. Wei W, Zheng-zhong B, Yong-jie W, Qing-wu Z, Ya-lin M. Bioeffects of low-frequency ultrasonic gene delivery and safety on cell membrane permeability control. J Ultrasound Med 2004; 23: 1569-82.

22. Franco de Oliveira R, Pires Oliveira DA, Soares CP. Effect of low-intensity pulsed ultrasound on 1929 fibroblasts. Arch Med Sci 2011; 7: 224-9.

23. Duvshani-Eshet M, Machluf M. Therapeutic ultrasound optimization for gene delivery: a key factor achieving nuclear DNA localization. J Control Release 2005; 108: 513-28.

24. Kim HJ, Greenleaf JF, Kinnick RR, Bronk JT, Bolander ME. Ultrasound-mediated transfection of mammalian cells. Hum Gene Ther 1996; 7: 1339-46.

25. Tata DB, Dunn F, Tindall DJ. Selective clinical ultrasound signals mediate differential gene transfer and expression in two human prostate cancer cell lines: LnCap and PC-3. Biochem Biophys Res Commun 1997; 234: 64-7. 